\title{
Modeling and Anti-skid Control of the Rail Vehicle Braking System
}

\author{
Wenliang Zhu ${ }^{1, a}$, Feng Diao ${ }^{2, b}$, Mengling $\mathrm{Wu}^{3, \mathrm{c}}$ \\ ${ }^{1,2,3}$ Institute of Railway and Urban Mass Transit, Tongji University, Shanghai, China \\ a 13661538012@126.com, b diaofeng698@163.com, cwuml_sh@163.com
}

Keywords: braking system modeling, PVU, anti-skid control, co-simulation, AMESim, Simulink Abstract. The performance of the braking system has an important influence on the safe operation of the train. In this paper, the AMESim simulation software is used to simulate the pneumatic valve unit (PVU) for the direct electro-pneumatic braking system controlled by the microcomputer. And the correctness of the model is verified by the simulation of the service braking and the emergency braking. At the same time, the vehicle dynamics model is built in the MATLAB/Simulink environment, and the AMESim pneumatic valve model is combined with the simulation to verify the effectiveness of the anti-skid logic. The simulation results from the service braking and emergency braking can be drawn that the PVU for the direct electro-pneumatic braking system controlled by the microcomputer is consistent with the response of the real system, which verifies the correctness of the PVU model. It can be seen from the simulation results that the anti-skid control logic can achieve the control requirements, and can achieve the stability of the anti-skid effect in the event of a continuous sliding. The results of this study provide an effective model base for the design of the actual vehicle braking system and solutions to the fault.

\section{Introduction}

At present, braking mode of railway vehicles is mainly dependent on the adhesion, but the maximum defect of the adhesion braking is the limited adhesion. The wheel /rail state has great influence on the wheel/rail adhesion. In the process of traction or braking, if the traction or braking torque is too large, wheels will idle or slide even lock. Therefore, it is necessary to control the braking force according to the different speed, load, environment and other conditions. The anti-skid system of railway vehicles is one of the effective means to solve the re-adhesion control during the train operation process. So it is necessary to carry out the simulation analysis and researches [1-2].

Frylmark and Johnsson used to model the existing anti-skid control methods by MATLAB/Simulink, and carried out the simulation evaluation [3]. It is considered that the method of noise spectrum analysis can be used to detect the adhesion coefficient between wheel and rail by Maksym Spiryagin. The relationship between the noise and the vehicle speed, the slip ratio, the adhesion coefficient can be obtained by the data from the inquiry study [4]. In order to improve the speed of the anti-skid control of railway vehicles, Hiro-o Yamazaki established the model of adhesion characteristics through a beam model [5]. Zheming Chen studied the anti-skid problem of air braking in the train [6]. According to the friction characteristics of the disc braking system and the change of the brake cylinder pressure, the vehicle braking dynamics model and the single wheelset dynamic model are established by Ren Luo [7]. The characteristics of pneumatic braking system and the selection of control parameters are analyzed by Jianyong Zuo [8]. 


\section{Working principle of braking system for rail vehicles}

The rail vehicle adopts the direct electro-pneumatic braking system controlled by the microcomputer which is mainly composed of braking instruction and transmission unit, braking control system (MBCU: microcomputer braking control unit), the pneumatic braking unit (PBCU: Pneumatic braking control unit), electric braking control unit, foundation braking equipment, anti-skid control unit, air supply unit and train pipes. The combined braking mode of electric braking and air braking is used to control a braking unit of a motor car and a trailer car. The structure principle is shown in Figure 1. The braking control device through the train control and management system (TCMS networks) receives braking instructions from the driver's braking controller or ATP to realize the braking force distribution, the electro-pneumatic braking and the anti-skid control.

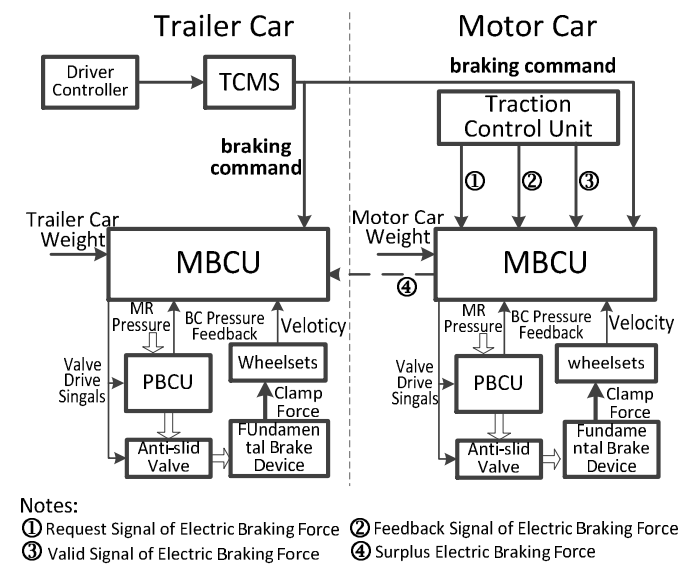

Fig. 1 Control principal diagram of the braking system (Take 1M1T for example)

\section{Working principle of the anti-skid control}

When the sliding is detected, the anti-skid control policy makes the brake cylinder exhaust or hold pressure. The main control strategy adopted at present is the anti-skid device which works according to the threshold of the speed difference, the deceleration and the slip rate. We can take a single criterion and also can integrate 3 criteria.

Based on the force analysis of the wheelset, the dynamic model of the air braking of the subway is established in MATLAB/Simulink. After applying the braking force, the velocity and the deceleration of an axle are calculated. Choose an axle velocity as the reference axle velocity $V_{\text {ref. }}$. The difference between the velocity of each axle $V_{n}(n=1,2)$ and the reference axle is the velocity difference $\Delta V_{n}(n=1,2)$.

According to the calculated axle velocity, deceleration and velocity difference between anti-skid criterion, we can make the sliding judgment. When the anti-skid criterion is satisfied, the anti-skid unit is controlled by the anti-skid valve or the anti-skid valve will exit sliding control.

The anti-skid control strategy taken in this paper is shown in Table 1. Once the sliding judged, the hold valve will obtain electricity and the air intake channel of the brake cylinder will be cut off. The exhaust valve of the sliding axle is turned on and then turned off after 50ms. At the same time, the brake cylinder pressure is in the state of holding pressure and continued to 50ms. During the period of holding pressure, the anti-skid device continues to judge the sliding. If the axle stops sliding, the anti-skid unit stops controlling. Otherwise the exhaust valve carries out the second exhaust for $50 \mathrm{~ms}$ and holds the pressure for $50 \mathrm{~ms}$, and the like. When the holding time is more than 
$8 s$ or the duration of exhaust time is more than $4 s$, turn off the anti-skid control to ensure the braking ability.

Table 1 Anti-skid control strategy

\begin{tabular}{ccc}
\hline Exhaust frequency & Air exhaust valve opening time $/ m s$ & Air exhaust valve closing time $/ m s$ \\
\hline 1 & 50 & 50 \\
2 & 50 & 50 \\
3 & 150 & 50 \\
4 & 250 & 50 \\
5 & 4000 & \\
\hline
\end{tabular}

\section{The pneumatic valve unit and single wheel braking dynamics modeling}

\section{Air braking system modeling}

The air braking subsystem of the direct electro-pneumatic braking system controlled by the microcomputer mainly includes EP valve, relay valve, emergency valve, empty and load adjustment valve, anti-skid valve, brake cylinder, braking lines, and so on [9]. Emergency valve, anti-skid valve can be simulated by using universal solenoid valve of AMESim. EP valve, relay valve, empty and load adjustment valve need the secondary development of AMESim. EP valve is one of the key component of the electro-pneumatic braking system. Its function is to adjust the output pressure of the relay valve volume chamber according to the braking control command. At present, switch-type EP valve is the most commonly used in the field of vehicle braking. It's mainly composed of braking solenoid valve, relief valve, pressure sensor, volume chamber, controller, and so on [10], as is shown in Figure 2. Its working principle is that when the braking instruction is obtained, the braking solenoid valve is electrically powered, and the valve port is opened. The pre-control pressure passes to the back part by the EP valve and at the same time the pressure sensor feeds back the pre-control signal. If the pressure signal is below the command value, the braking solenoid valve is electrically charged. If it is higher than the instruction value, then the release electromagnetic valve is relieved to discharge, until the pressure signal reaches the instruction value. We can realize the control of increase or decrease of the pre-control pressure by turning on or off the braking solenoid valve and relief solenoid valve, and finally make the target brake cylinder pressure meet the braking requirements. Due to the volume effect and gas flow inertia, the switch-type EP valve is controlled by the basic switch, and the dynamic response and the control precision cannot meet the requirement of braking. The PWM signal is mostly used in actual engineering control. This paper uses the PID feedback control method and establishes the EP valve in AMESim, as shown in Figure 2(a). The EP valve model has ideal dynamic response and control precision. We encapsulate it by using the function of super element in AMESim, as shown in Figure 2(b). The other pneumatic valves and components models are established by using the similar method, and the gas path information is connected with the pipeline connection between the pneumatic components by AMESim. Air braking system model of a motor car and a trailer car is shown in Figure 3. Using the method of literature [9], the model validation of the air braking system is carried out, as shown in Figure 4. The validation results show that the model can be used to simulate the air braking subsystem. 


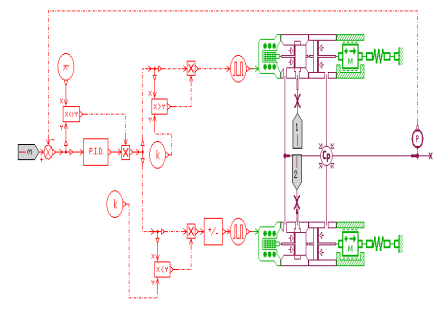

(a) simulation model

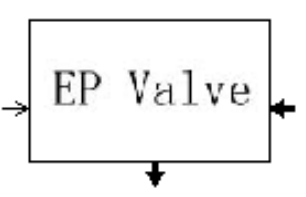

(b) Super component model

Fig. 2 Operation principle of EP valve and simulation model based on AEMSim

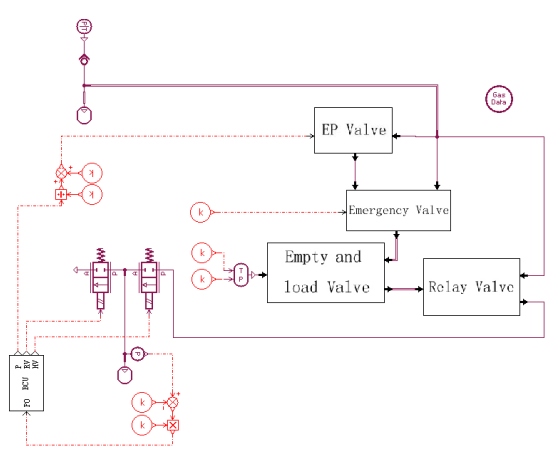

Fig. 3 Pneumatic braking subsystem based on AMESim

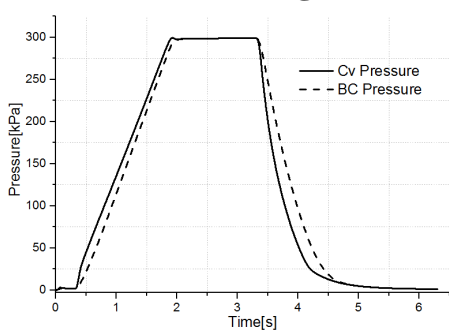

(a) Service braking

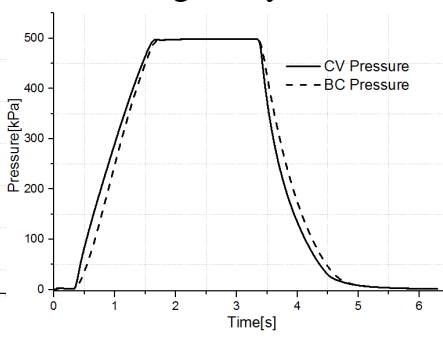

(b) Emergency braking

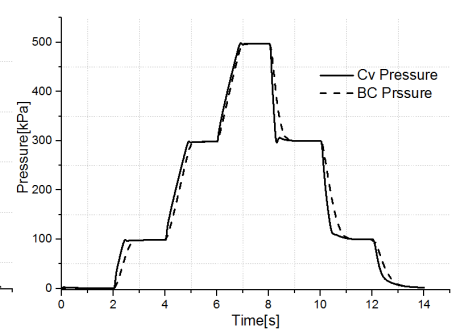

(c) Stage braking

Fig. 4 Model validation of pneumatic braking subsystem

Braking dynamics simulation model of a single wheel

The force state of the wheel under the braking condition is shown in Figure 5. According to Figure 5, the dynamic equation of the wheel rotation is as following:

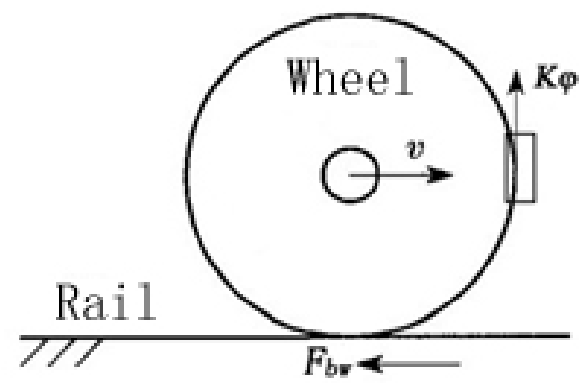

Fig. 5 Force diagram of the wheelset under the braking condition According to Figure 5, the equation of dynamics of a single wheel is established:

$$
\begin{aligned}
& \frac{d v}{d t}=- \frac{F_{F_{w}}}{\{n} \\
& \frac{d d}{d t}=\frac{\left(K \varphi-F_{b w} r\right)}{I_{w}}
\end{aligned}
$$


where $v$ is the longitudinal motion of a wheel, $F_{b w}$ is the longitudinal adhesion of wheel/rail, $m$ is the quality on wheels, $\omega$ is the angular velocity of wheels, $t$ is the time, $r$ is the wheel radius, $K$ is the wheel clamping force, $\varphi$ is the friction coefficient between tread and the brake-shoe, $I_{w}$ is the moment of inertia of wheelset. According to the above formula and parameters, the dynamic simulation model is established, which is divided into the speed simulation model and the wheel speed simulation model.

1.Vehicle speed simulation model

Figure 6 is the simulation model for vehicle speed. The input signal $F_{b w}$ is the longitudinal adhesion. $m$ is the $1 / 8$ train quality, and that is the quality on the wheel. $V_{0}$ is the initial velocity of train starting braking. The output signal $a$ is the deceleration of the train (the value is negative). $S$ is the braking distance. $V_{s}$ is the train velocity.

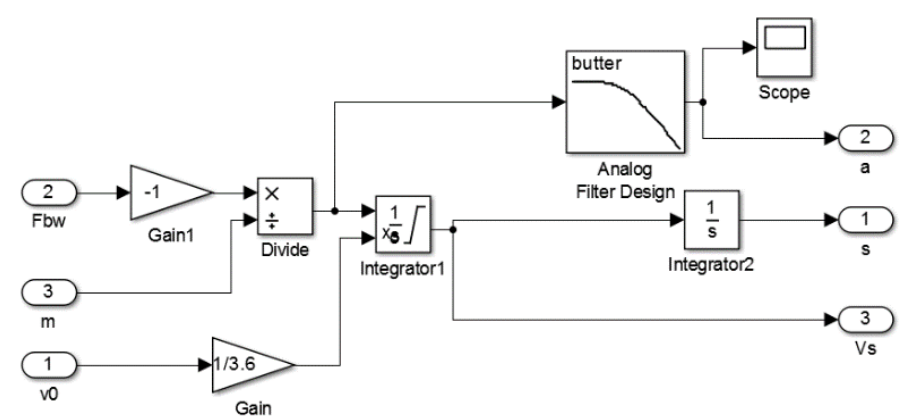

Fig. 6 Sketch map of vehicle speed simulation model

2.Wheel speed simulation model

Figure 7 is a simulation model of wheel speed. Input signal $K$ is the wheel clamping force. fai is the coefficient of friction between the brake-shoe and the wheel tread surface. $F_{b w}$ is the longitudinal adhesion and $r$ is the wheel radius. $V_{0}$ is the initial velocity of train starting braking and $I_{w}$ is the moment of inertia of the wheel. Output signal $V_{w}$ is the wheel speed of the train (wheel speed is equal to the angular velocity of the ride radius).

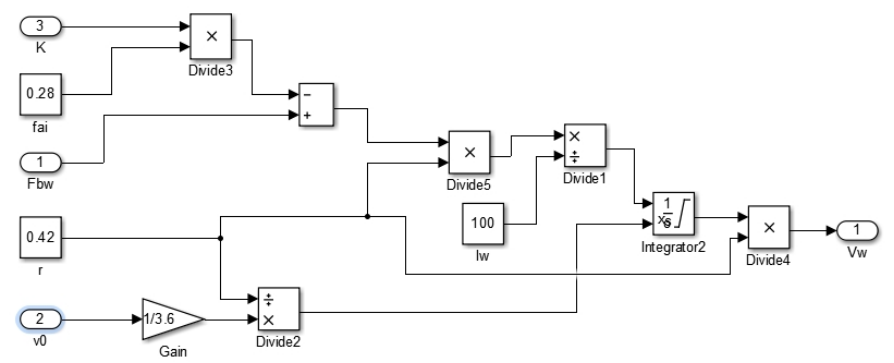

Fig. 7 Sketch map of the wheel speed simulation model 
The overall simulation model of the braking system is shown in Figure 8.

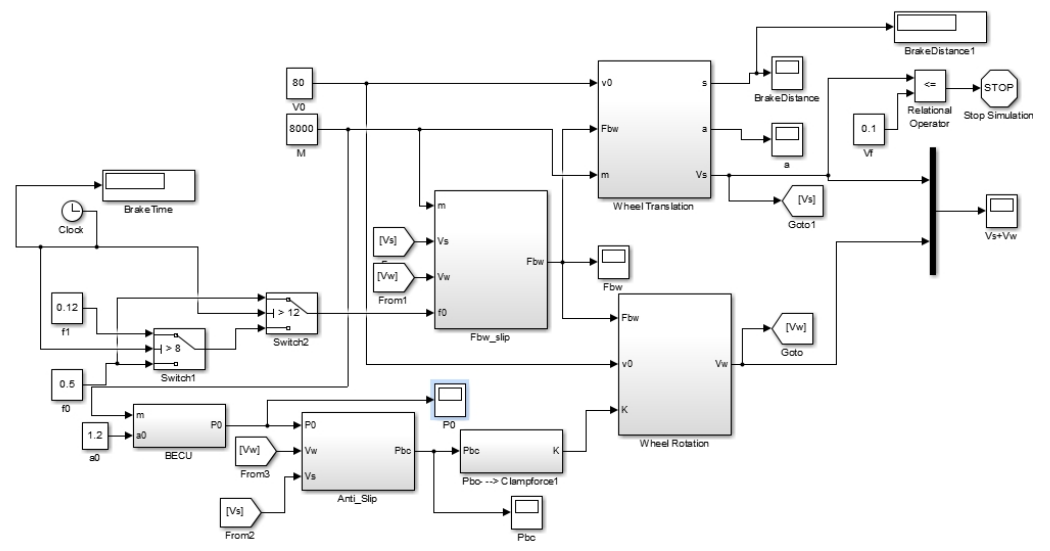

Fig. 8 The whole simulation model of the braking anti-skid control system

\section{Anti-skid control simulation}

During the whole braking progress from the start to the end, we do the simulation under the condition that the initial velocity is $80 \mathrm{~km} / \mathrm{h}$. In this process, the dynamic data of each parameter can be simulated and the regularity of these parameters with the change of braking time is drawn. The dynamic performance parameters can be obtained: braking time and braking distance. Data need to be analyzed are : changes of brake cylinder pressure $P_{0}$ and the control signal of hold valves and exhaust valves, the relationship between the vehicle speed $V_{s}$ and the wheel speed $V_{w}$, changes of the acceleration (deceleration) $a$, changes of the slip ratio $\sigma$ and the adhesion coefficient $\mu$.

Figure 9 and Figure 10 is a graph of the slip ratio and adhesion coefficient which change with the braking time in the anti-skid control process. It can be seen from the chart that the slip rate has an obvious rising process, and the adhesion coefficient decreases at the beginning. However, due to the installation of anti-skid control system, the slip ratio gradually declines, and the adhesion coefficient gradually picks up. When the slip ratio drops close to zero, the system decides that it was not in the sliding, so the slip ratio and adhesion coefficient repeat changes as before. It is worth noting that although the magnitude of the slip ratio in the repeated oscillation, but the amplitude of the oscillation is increased, until the end of the braking. Both the vehicle speed and the wheel speed are 0 , the slip rate is 1 .

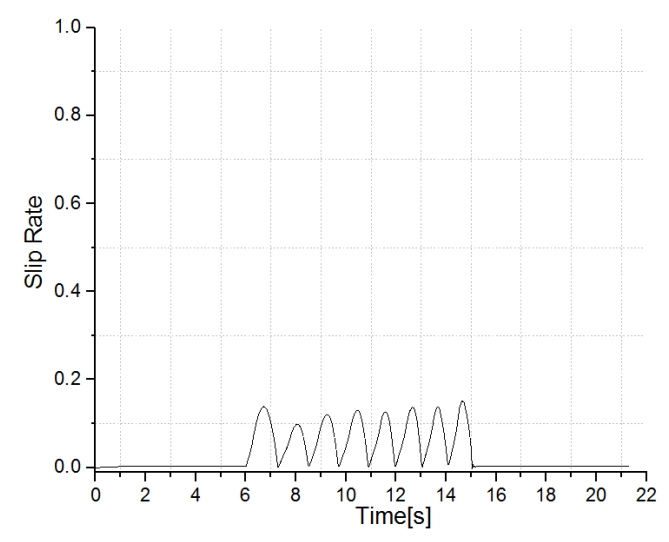

Fig. 9 Slip ratio curve

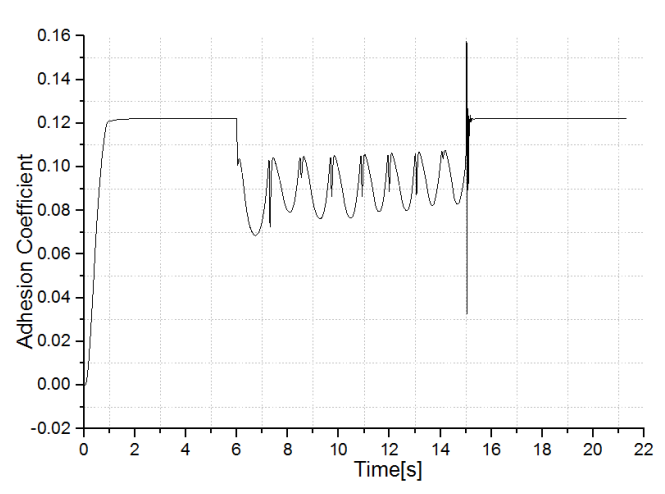

Fig. 10 Adhesion coefficient curve

The change of acceleration is similar. At the beginning of the braking, acceleration is achieved in a short time to achieve the goal of pre-set acceleration $a_{0}$. When the sliding begins, wheels get 
locked and the acceleration decreases. The anti-skid system judges the occurrence of sliding, then the anti-skid valve works. The brake cylinder pressure makes corresponding changes, so that the acceleration gradually picked up to close to the target acceleration $a_{0}$. And then the anti-skid system releases the sliding decision, but at this time the train is still in a state of sliding. So the wheel speed begins to decrease, the antiskid system subsequently makes the action as before and cycles until the end of braking. The changes are shown in Figure 11.

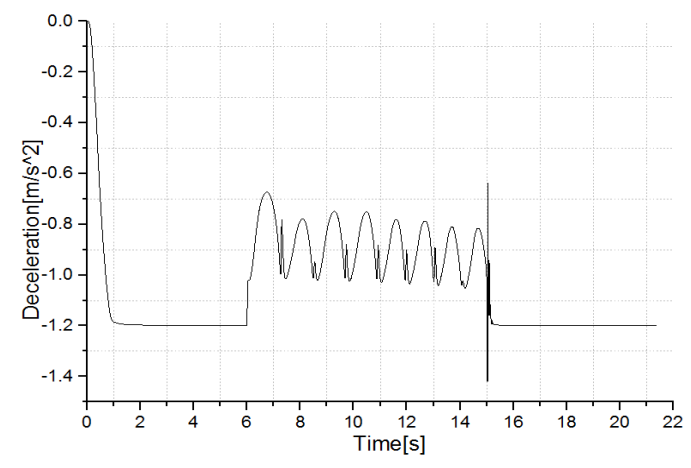

Fig. 11 Acceleration curve

The reason for the change of the train speed curve is also similar. Before sliding, the wheel speed is basically the same as the vehicle speed, and both are in steady decline. At the beginning, due to the change of the acceleration, the wheel speed drops, and the anti-skid system judges that the wheel starts to slide, then the anti-skid valve and the brake cylinder make the corresponding action, so that the wheel speed will be back to the same as the vehicle speed. Afterwards, the wheel speed changes according to the change of acceleration. The curve of the vehicle speed and the wheel speed changing with the braking time is shown in Figure 12.

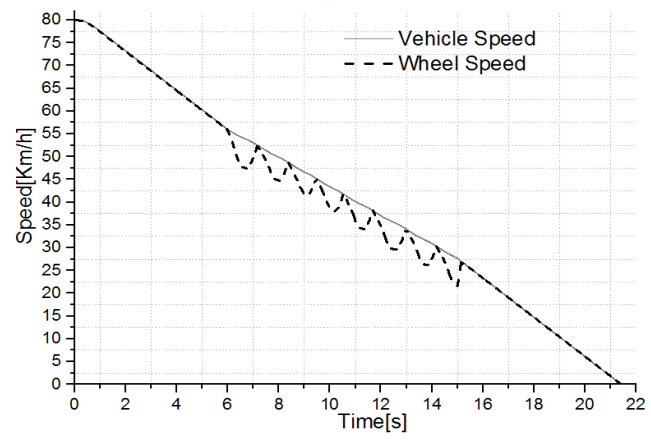

Fig. 12 Vehicle speed curve

The reason why the acceleration and the speed change is that the anti-skid valve controls the brake cylinder to make the corresponding movement. The control signals of the hold valve and the exhaust valve of the anti-skid valve are shown in Figure 13 and Figure 14.

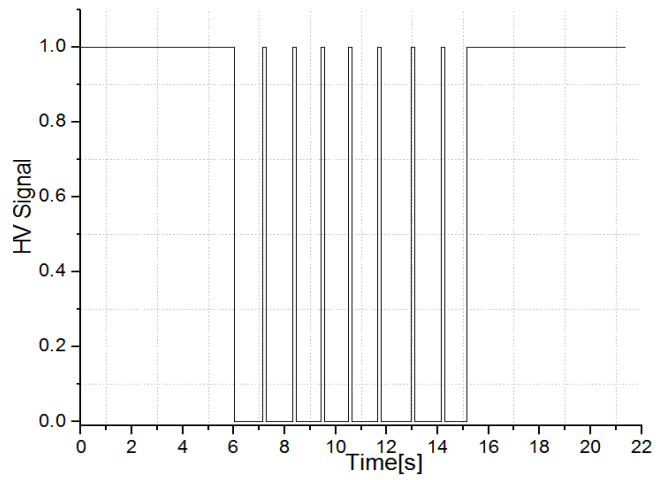

Fig. 13 Hold valve control signal

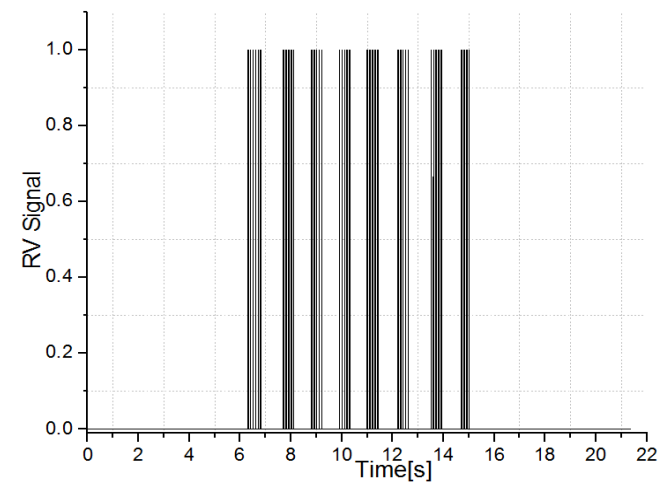

Fig. 14 Exhaust valve control signal 
The brake cylinder pressure changes with the braking time as shown in Figure 15. Before the sliding occurrence, the anti-skid valve works in the inflation position, and the brake cylinder pressure is always maintained as the target pressure $P_{0}$. At the beginning of the slide $(8 \mathrm{~s}$ in the diagram), the exhaust valve was electric, and the pressure of the brake cylinder was reduced. When the speed is gradually restored, the slide has been alleviated ( $9 s$ in the diagram). The hold valve and the exhaust valve are not electric and maintain the pressure at this time, the pressure of the brake cylinder remains unchanged. When the slip is relieved and the decision of the slip is lifted (10s in the diagram), the pressure protection valve is electrically charged, and the brake cylinder begins to inflate, then the brake cylinder pressure is restored to the target pressure. Hold valve, exhaust valve and brake cylinder operation are also the case of the cycle until the end of the braking in the rest of the time.

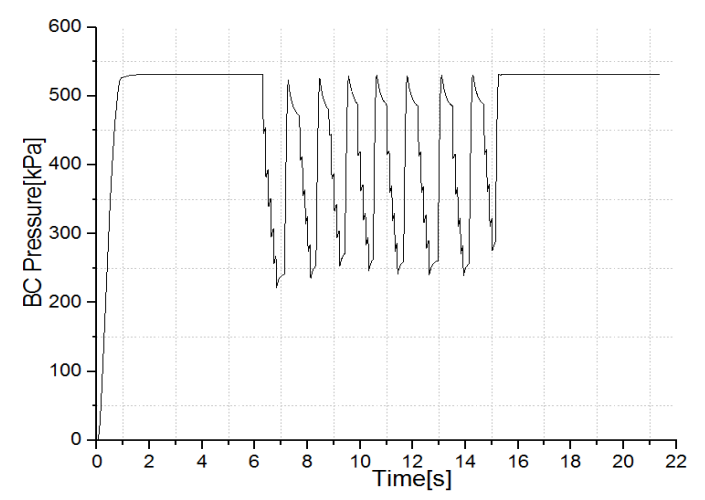

Fig. 15 Brake cylinder pressure change curve

\section{Conclusion}

In the paper, we use AMESim to simulate the gas path of the direct electro-pneumatic braking system controlled by the microcomputer, use MATLAB/Simulink to build anti-skid control system and combine AMESim to realize the co-simulation to verify the validity of anti-skid logic. The simulation results can be drawn that the PVU is consistent with the response of the real system. The anti-skid logic can meet the control requirements of the actual system, and can be applied to the design of the actual vehicle braking system and the solution of the fault.

\section{Acknowledgements}

This project is financially supported by National Natural Science Foundation of China (U1534205) and Key Projects in the National Science \& Technology Pillar Program during the Twelfth Five-year Plan Period (2015BAG12B01).

\section{References}

[1] Suh M W, Park Y K, Kwons J. Braking performances simulation for a tractor-semitrailer vehicle with an air brake system [J]. Journal of Automobile Engineering, 2002, 216(1):43-54.

[2] Chol J J, Park S H, Kim J S. Dynamic adhesion model and adaptive sliding mode brake control system for the railway rolling stocks [J]. Journal of Rail and Rapid Transit, 2007, 221(3):313-320.

[3] D,Frylmark, S.Johnsson. Automatic slip control for railway vehicles [D]. Linköping, Sweden: Linköpings Universitet, 2003. 
[4] Maksym Spiryagin, Kwan Soo Lee, Hong Hee Yoo. Control system for maximum use of adhesive forces of a railway vehicle in a tractive mode [J]. Mechanical Systems and Signal Processing, 2008, 22 (3): 709-720.

[5] Hiroo YAMAZAKI, MasaoNAGAI, Takayoshi KAMADA. A Study of Adhesion Force Model for Wheel Slip Prevention Control [C]. JSME International Journal, 2004, 47 (2): 496-501.

[6] Zheming Chen, Jing Zeng, Ren Luo. Anti-skid Control and Simulation of Air Braking [J]. Railway Transaction, 2009 (4):25-31. (In Chinese)

[7] Ren Luo, Jing Zeng. Simulation of Railway Vehicle Anti-skid Control [J]. Journal of Mechanical Engineering, 2008 (3):29-34. (In Chinese)

[8] Jianyong Zuo, Lihui Ren, Mengling Wu. Simulation and Experimental Study on Anti-skid control of Railway Vehicle Braking System [J]. Journal of Tongji University, 2010, 38(6):912-916. (In Chinese)

[9] Jianyong Zuo, Zongming Wang, Mengling Wu. Simulation Model of Air Braking System for Subway Train [J]. Journal of Traffic and Transportation Engineering, 2013, 13(2):42-47. (In Chinese)

[10] Jiang Liu, Meichuan Yang, Hailong Xia. Simulation Research on the On-off EP Valves Based on AMESim [J]. Rolling Stock, 2014, 52(4):12-15. (In Chinese) 